\title{
Investigation of new volumetric non-destructive techniques to characterise additive manufacturing parts
}

\author{
A-F. Obaton ${ }^{1}$ (D) M-Q. Lê ${ }^{2} \cdot$ V. Prezza ${ }^{3} \cdot$ D. Marlot ${ }^{3} \cdot$ P. Delvart $^{3} \cdot$ A. Huskic $^{4} \cdot$ S. Senck ${ }^{4} \cdot$ E. Mahé $^{1} \cdot$ C. Cayron $^{1}$
}

Received: 7 June 2017 / Accepted: 6 April 2018 / Published online: 5 June 2018

(C) The Author(s) 2018

\begin{abstract}
The assessment of the potential of various non-destructive methods to characterise additive manufactured dense and lattice parts was investigated. The Archimedes' and gas pycnometric methods for density measurements of both types of structures are presented as well as the percentage of cells in lattice structures. A multi-frequency eddy current method for electrical conductivity measurements of lattice structures is also presented. Finally, C-scan ultrasound method for the characterisation of dense parts was investigated. The advantages and limitations of each method are underlined.
\end{abstract}

Keywords Additive manufacturing · Volumetric non-destructive techniques (NDTs) · Archimedes' method · Gas pycnometric method $\cdot$ Multi-frequency eddy current method $\cdot$ C-scan ultrasound method

\section{Introduction}

In contrast to traditional manufacturing techniques, additive manufacturing (AM) is a technology which enables the fabrication of very complex geometry parts (internal structures and

This article is part of the Topical Collection on Welding, Additive Manufacturing and Associated NDT

\section{A-F. Obaton}

anne-francoise.obaton@lne.fr

M-Q. Lê

sciensoria@gmail.com

V. Prezza

contact@eurosonic.com

A. Huskic

Sascha.Senck@fh-wels.at

1 Laboratoire commun de métrologie (LNE/Cnam), Laboratoire National de Métrologie et d'Essais (LNE), 1 rue Gaston Boissier, 75015 Paris, France

2 Sciensoria, 35170 Bruz, France

3 Mistras Group, Eurosonic, 94370 Sucy-en-Brie, France

4 University of Applied Sciences Upper Austria, Stelzhamerstraße 2, 4600 Wels, Austria lattice structures). Furthermore, AM facilitates the mass production of highly customised parts, e.g., in relation to individualised medical implants. Considering these two points, characterisation techniques which were formerly used for parts manufactured with conventional methods have to be reconsidered. New non-destructive techniques (NDT) [1] as well as volumetric techniques have to be investigated. $\mathrm{X}$-ray tomography is presently the most appropriate technique [2-4] considering that it is a non-contact technique providing a high spatial resolution. However, it is a costly and time-consuming technique which may be unsuitable for mass production.

The French National Metrology Institute, LNE, in collaboration, with NDT companies, Sciensoria and Eurosonic, have investigated two of the new NDT technologies that they are proposing: multi-frequency eddy current and C-scan ultrasound respectively. In parallel, at LNE, we are applying density measurements to characterise the repeatability as well as the reproducibility of AM parts. This quantity is measured by two different methods: Archimedes' and gas pycnometric methods. For a first exploration of these four methods, several dense specimens with different a priori defined defects and lattice specimens were studied.

In this paper, we will present the measurement outcomes of these AM specimens characterised by the above-mentioned methods, compare the results, and highlight the advantages and the limitations of each method. 


\section{Density and percentage of lattice cell measurements}

\subsection{Archimedes' method}

\subsubsection{Measurement system}

The density of the specimens was determined by the hydrostatic method at LNE, the National Metrology Institute (NMI) in France. The system (Fig. 1) consists of a precision balance with an inferiorly attached thin wire at the end of which a suspension device is hooked. This suspension device is immersed in a container, filled with a liquid, placed beneath the balance. The balance used is of 400-g maximum weight capacity and of 0.1 $\mathrm{mg}$ resolution. The entire setup is placed in an air-conditioned laboratory at a temperature of $20 \pm 1{ }^{\circ} \mathrm{C}$ and at a humidity of 50 $\pm 10 \%$. The LNE density measurements, with this hydrostatic method, are traceable to the International System of units (SI).

\subsubsection{Measurement protocol}

The classical principle of the hydrostatic method involves measuring the apparent mass of the sample in air, then in twice-distilled water to deduce its density. However, in the case of lattice structures, in water, bubbles form at the interface air-lattice preventing the water to penetrate deep inside the lattice because the surface tension of water is too high $\left(72.8 \times 10^{-3} \mathrm{~N} \mathrm{~m}^{-1}\right.$ at $\left.20{ }^{\circ} \mathrm{C}\right)$. In order to avoid this phenomenon, which distorts the measurements, absolute ethanol was used. In that case, a good penetration of the liquid into the

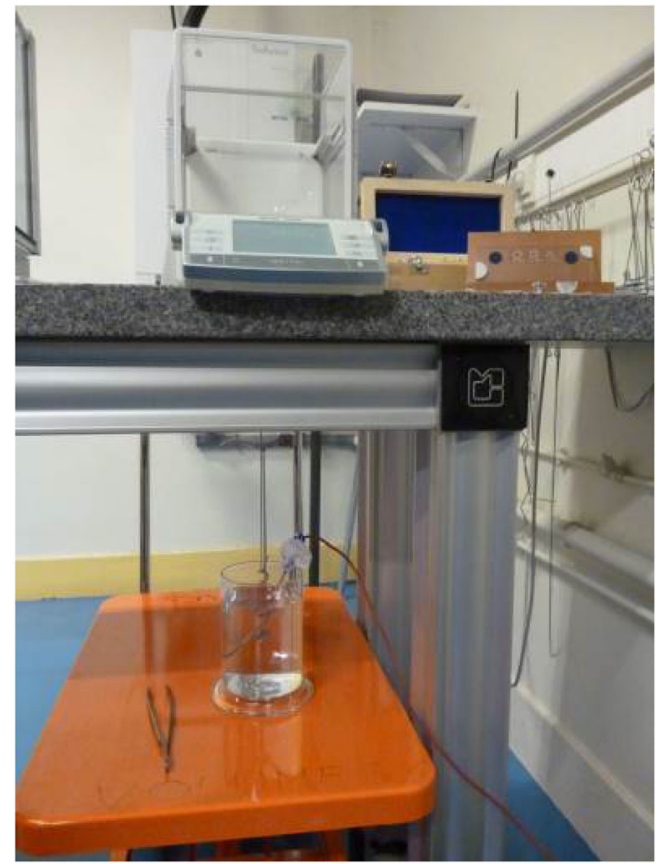

Fig. 1 LNE Archimedes' method setup used to measure the studied parts in density lattice structure was observed as the surface tension of alcohol $\left(22.27 \times 10^{-3} \mathrm{~N} \mathrm{~m}^{-1}\right.$ at $\left.20^{\circ} \mathrm{C}\right)$ is much lower than the one of water, preventing bubbles to disturb the measurement.

The samples were placed in the $20^{\circ} \mathrm{C}$ air-conditioned laboratory 1 day before measurements. So, its temperature is assumed to be $T_{0}=20^{\circ} \mathrm{C}$. Then, the below operating mode was followed:

1. The balance was tared to zero.

2. Weighing in air $\left(P_{a}\right)$ : the sample was placed on the balance and compared with mass standards according to Borda's double-substitution method.

3. The density of air $\left(\rho_{a}\right)$ was calculated taking into account the temperature $\left(T_{a}\right)$, the pressure, and the humidity in the laboratory during measurements of the sample in air.

4. The sample was removed from the balance.

5. An eventual derivation of the tare was checked. If a derivation is observed, the weighing is done again.

6. The balance is tared to zero

7. Weighing in absolute ethanol $\left(P_{l}\right)$ : the sample was placed in the absolute ethanol on the suspension device. Once the sample and the temperature were stabilised, the mass of the sample was compared with mass standards placed on the weighing pan according to Borda's doublesubstitution method. This comparison in absolute ethanol was carried out twice. In between each measurement, an eventual derivation of the tare was checked and then the balance is tared to zero if no derivation is observed.

8. The temperature of the absolute ethanol inside the container was taken $\left(T_{l}\right)$ in order to take into account the variation of temperatures on the density of alcohol. Indeed, the density of the ethanol $\left(\rho_{l, T_{0}}\right)$ was preliminary evaluated by a pycnometric method at the reference temperature of $T_{0}=20^{\circ} \mathrm{C}$.

9. The density of air $\left(\rho_{a, l}\right)$ was calculated taking into account the temperature $\left(T_{a}\right)$, the pressure, and the humidity in the laboratory during measurements of the sample in ethanol.

10. Weighing in air $\left(P_{a}\right)$ : the sample was placed for the second time on the balance and compared with mass standards according to Borda's double-substitution method.

11. The final result $(\rho)$ is expressed as the mean of the two measurements carried out using the following relation:

$\rho=\frac{A_{a}}{A_{a}-A_{l}}\left\{\rho_{l, T_{0}}\left[1+C_{l}\left(T_{l}-T_{0}\right)\right]\left[1+C_{s}\left(T_{l}-T_{0}\right)\right]\right.$
$\left.-\rho_{a}\left[1+C_{s}\left(T_{a}-T_{0}\right)\right]\right\}+\rho_{a}\left[1+C_{s}\left(T_{a}-T_{0}\right)\right]$

where

$A_{a}=P_{a}\left(1-\frac{\rho_{a}}{\rho_{e}}\right)$ and $A_{l}=P_{l}\left(1-\frac{\rho_{a, l}}{\rho_{e}}\right)$ 
with $C_{l}$ and $C_{s}$ the thermal expansion coefficient of the liquid and of the sample respectively and $\rho_{e}=8000 \mathrm{~kg} \mathrm{~m}^{-3}$, the conventional value of the density of the standards.

This equation takes into account the air buoyancy correction.

The expanded uncertainty on measurement is calculated using a coverage factor $k=2$, taking into account the following sources of uncertainty:

\section{- Balance}

- Mass standards

- Comparisons with mass standards

- Density of the water

- Density of the air

- Volume expansion of the solid

- Reproducibility of the measurements

Then, comparing the measured volume by the hydrostatic method with the one measured with a digital calliper, the percentage of material relative to the lattice was also determined.

\subsubsection{Results}

The measurements of 30 AM cubic dense specimens in TiAl6V, characterised by internal pores that are increasing in size for consecutive specimen ( 20 specimens) and a second batch with internal canals that as well are increasing in diameter (ten specimens), were performed (see Figs. 2 and 3). For each pore and canal diameter, two specimens with the same feature diameter were manufactured using a Concept Laser M2 Cusing.

As can be observed from Figs. 2 and 3, the AM process is not repeatable as two similar specimens have different densities. Furthermore, the density should decrease as the size of the pores and canals are decreasing. This is not the case so the parts are not in compliance with the specifications and the process has to be reviewed. Other samples will be manufactured with a reviewed process in order to gain repeatability and confidence into the AM process.

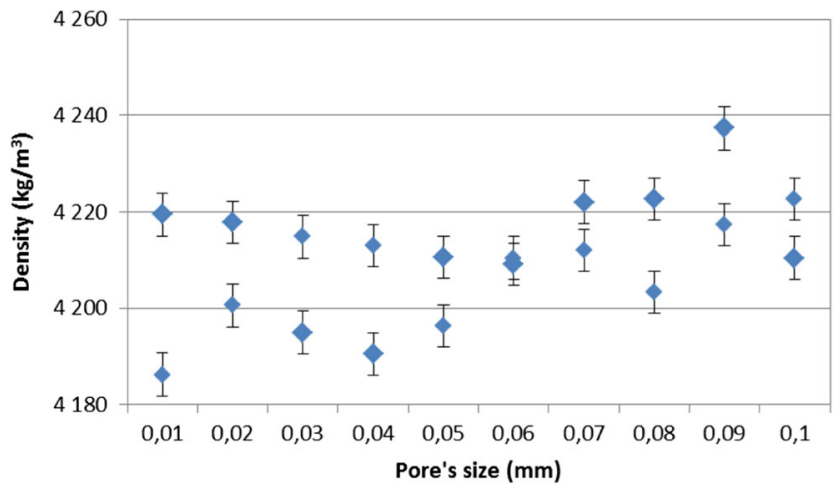

Fig. 2 Density measurements of dense specimens with different sizes of inside pores using an Archimedes' method

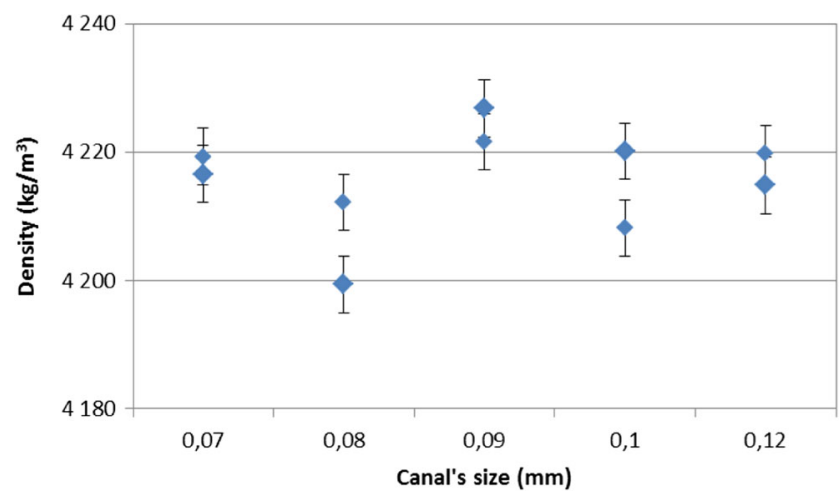

Fig. 3 Density measurements of dense specimens with different sizes of inside canals using an Archimedes' method

Additionally, measurements of cubic lattice specimens in TiAl6V, with different percentage of cells: 77 and $79 \%$ such as given by the initial part specifications (two identical specimens per percentage), were performed (Figs. 4 and 5).

All these specimens were fabricated during the same AM process and as can be shown in Fig. 4, their density is within the uncertainty bars so one can conclude that the AM process is repeatable. Figure 5 gives the evaluation of the percentage of lattice cells. The difference in the percentage of lattice cells is noticeable in between two different specimens and the repeatability of the AM regarding this aspect process seems good. There is an offset from 2 to $4 \%$ in between the theoretical and effective percentage of lattice cells.

\subsubsection{Benefits and limitations of the method}

The Archimedes' method enables to perform density measurements of dense structures but also of lattice structures with a good accuracy. It also provides the percentage of lattice cells. The knowledge of these two quantities allows verifying the compliance with the part specifications. Furthermore, comparing the density of an AM part with the density of a part fabricated by classical methods with the same material provides a

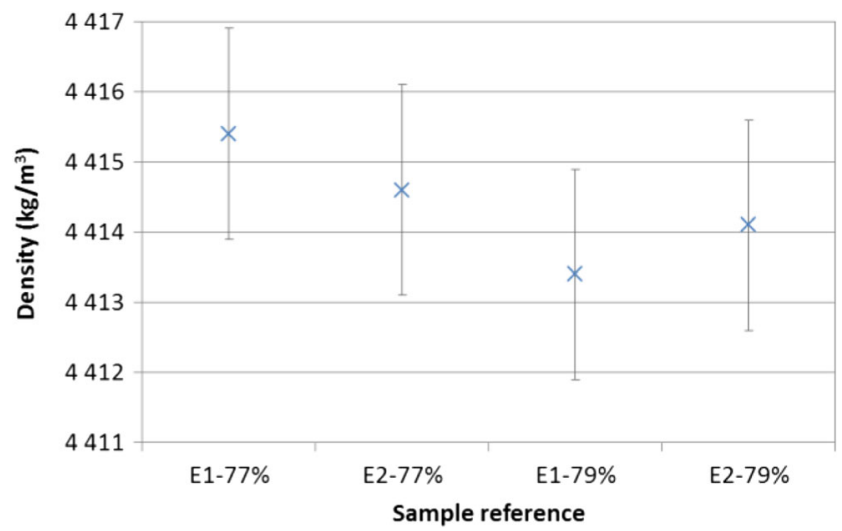

Fig. 4 Density measurements of lattice specimens with different theoretical cell sizes (77 and 79\%) using an Archimedes' method 


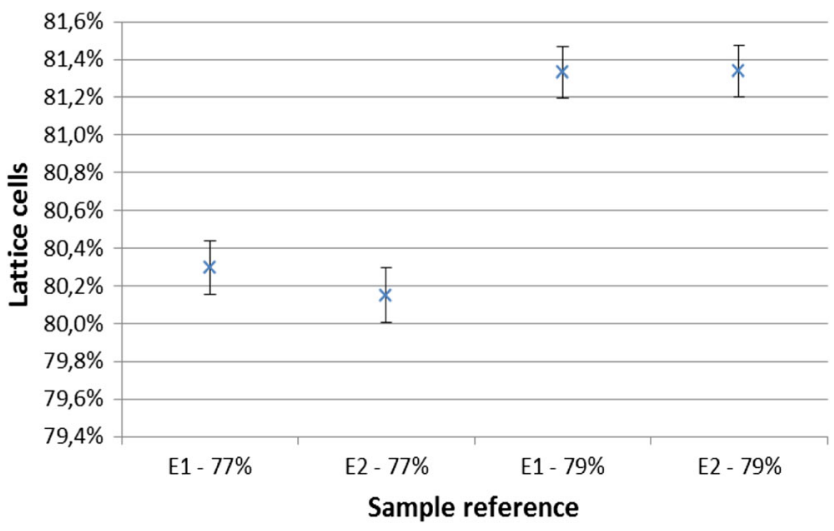

Fig. 5 Percentage of cells in lattice specimens with different theoretical cell sizes (77 and $79 \%$ ) extracted from measurements using an Archimedes' method

quantification of the material in terms of internal porosities. Moreover, comparing similar parts fabricated during the same AM process and similar parts fabricated in different AM processes enable to study the repeatability and the reproducibility, respectively, of the AM processes. However, the measurements turn out to be rather time-consuming ( $20 \mathrm{~min}$ per part).

\subsection{Gas pycnometric method}

The density of the same cubic lattice specimens in TiAl6V, with different percentage of cells, measured previously by the Archimedes's method, was also evaluated by the gas pycnometric method.

\subsubsection{Measurement system}

Since gas pycnometer measures volumes to evaluate the density, a weighing of the part needs to be performed. The LNE gas pycnometer used for volume measurements is an Ultrapyc 1200e from Quantachrome Instrument (Fig. 6). The same precision balance as the one used in the Archimedes' method was used. The pycnometer and the balance are localised in an airconditioned laboratory at a temperature of $20^{\circ} \mathrm{C} \pm 1{ }^{\circ} \mathrm{C}$. This pycnometer consists of two cells: a sample cell and an expansion cell which are connected to each other via a pipe which can be closed by a valve.

This system is provided with three sample cells. The larger one of $135 \mathrm{~cm}^{3}$ in volume (with an internal diameter of $49 \mathrm{~mm}$ and an internal depth of $75 \mathrm{~mm}$ ) was used for the present measurements as well as nitrogen gas.

The principle of the density measurement with a gas pycnometer is the following:

1. The device under test is placed in the pycnometer sample cell at the initial pressure of $P_{\mathrm{atm}}$. The valve is closed.

2. The pressure is increased until $P_{1}$ in the sample cell.

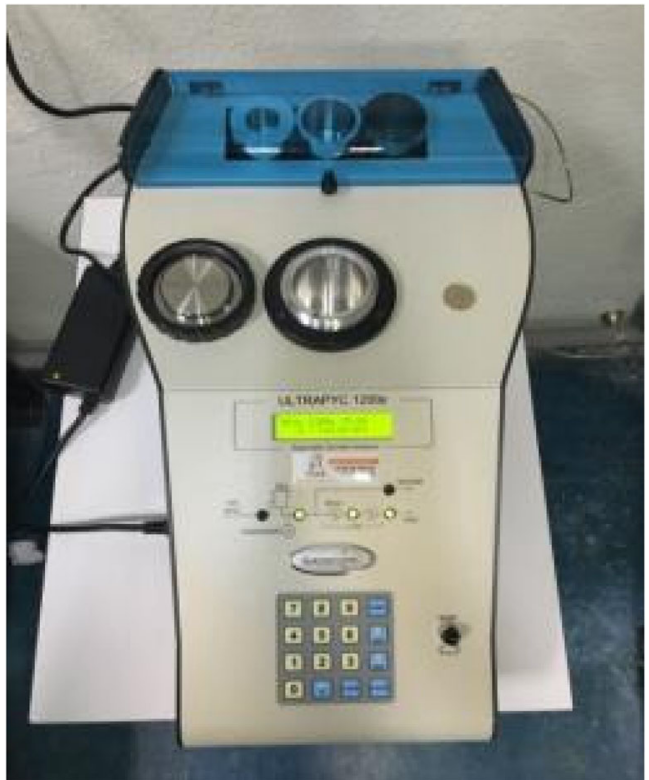

Fig. 6 LNE pycnometer used to measure the studied parts in volume

3. The pycnometer valve is open.

4. The pressure $P_{2}$ is measured when the equilibrium between the two cells is reached.

5. The apparent mass of the sample $(m)$ is measured with a precision balance.

6. The density is then calculated using the following equation:

$\rho=\frac{m}{V_{\text {sample }}+V_{\text {expansion }}\left(\frac{P_{2}-P_{\text {atm }}}{P_{2}-P_{1}}\right)}$

where $V_{\text {sample }}$ and $V_{\text {expansion }}$ are the volume of the sample cell and of the expansion cell respectively.

\subsubsection{Measurement protocol}

Before the pycnometric measurements, the cells are calibrated in volume with calibration spheres. Then, ten successive measurements, at a pressure $P_{1}$ of about $225 \mathrm{kPa}$, and with a 5 -min purging time, are performed. Finally, the sample is weighted.

\subsubsection{Results}

The measurements of the cubic lattice specimens are displayed in Fig. 7 and the extracted percentage of cells are showed in Fig. 8. These results are compared to the one performed above with the Archimedes' method.

As can be shown in Fig. 7, the variation of the pycnometric measurements agrees with the ones performed with the Archimedes' method. Nevertheless, the estimated 


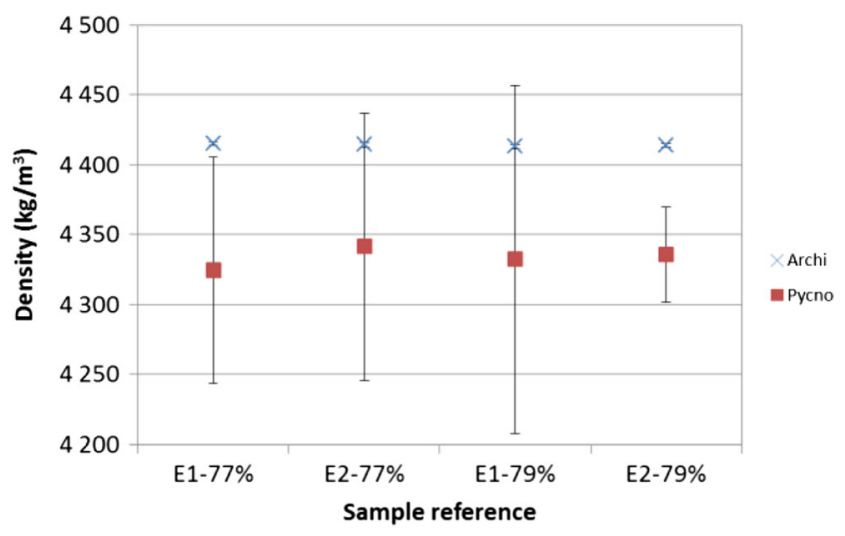

Fig. 7 Density measurements of lattice specimens with different theoretical cell sizes (77 and 79\%) using an Archimedes' and a gas pycnometric methods

uncertainties on the pycnometric measurements are under evaluated and have to be reviewed to be in accordance with the standard Archimedes' method.

\subsubsection{Benefits and limitations of the method}

The gas pycnometric method enables to perform volume measurements of dense structures but also of lattice structures. Then, associated with mass measurements, the density can be calculated. The percentage of lattice cells can also be provided. The knowledge of these two quantities allows verifying the compliance with the part specifications. Furthermore, comparing the density of an AM part with the density of a part fabricated with the same material by classical methods provides a quantification of the material in terms of internal porosities. Moreover, comparing similar parts fabricated during the same AM process and similar parts fabricated in different AM processes enables to study the repeatability and the reproducibility, respectively, of the AM processes. Finally, in comparison to the Archimedes' method, the measurements

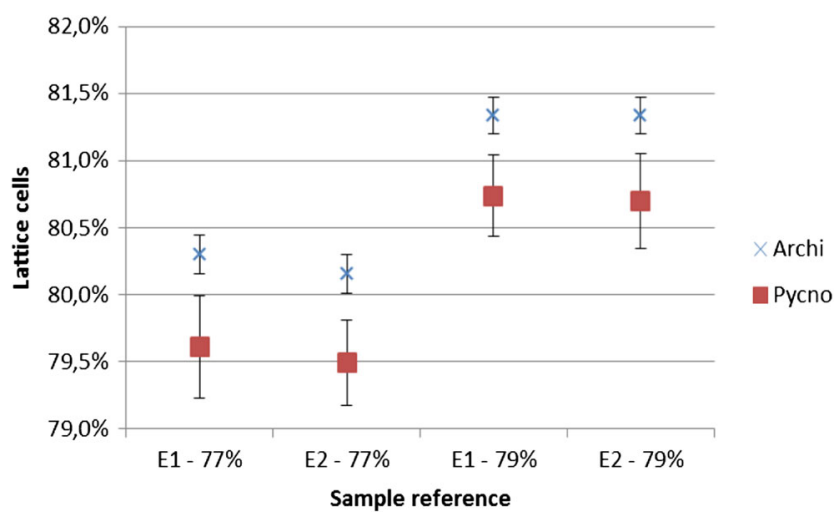

Fig. 8 Percentage of lattice specimens with different theoretical cell sizes (77 and 79\%) extracted from measurements using an Archimedes' and a gas pycnometric methods using the gas pycnometer are much faster, so the method is convenient for routine control. However, the uncertainty on measurement is not as good as with the Archimedes' method and the sample cell of the pycnometer is small (around $135 \mathrm{~cm}^{3}$ ) which prevents measurements of larger parts.

\section{Multi-frequency eddy current method}

The same cubic lattice specimens in TiAl6V, with different percentage of cells, measured previously by the Archimedes' and pycnometric methods, were also tested by an eddy current method.

\subsection{Measurement system}

The Sciensoria multi-frequency eddy current system ZScope*7 is presented in Fig. 9. It includes a transducer coupled to a tactile screen which displays the measurements. It can be connected to a computer to visualise the results on a dedicated software.

The transducer is configured in emitter/receiver mode and operates on a wide frequency bandwidth $(10 \mathrm{kHz}-40 \mathrm{MHz})$. The computer software performs analysis on the received signal and displays the complex impedance plane $Z=R+j X$. Each $Z$ curve represents several single measurements performed on a sample at a different distance. The higher the slope of a curve, the higher the electrical conductivity of the sample. However, the curves are not completely linear; consequently, their slopes are not constant. The Sciensoria's computer software makes use of an advanced signal analysis technique to attribute a unique conductivity value to each curve with very high precision. The system enables non-contact measurements and measurements are independent of the probe position.

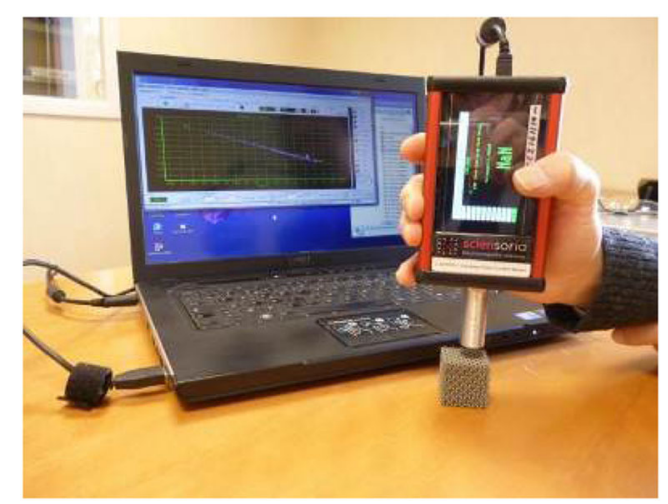

Fig. 9 Sciensoria eddy current system used to measure the studied parts in electrical conductivity 
Fig. 10 Three successive complex impedance measurements of two similar lattice specimens (E1 and E2) with a theoretical percentage of cells of $77 \%$ using an eddy current system

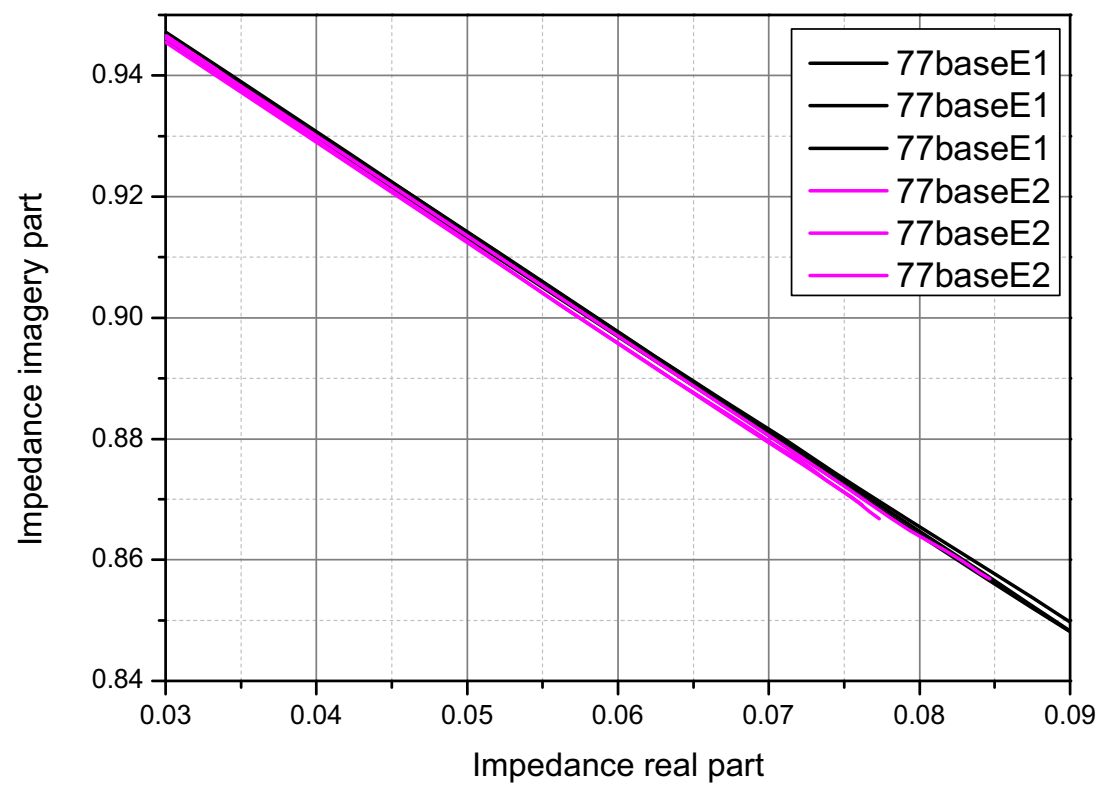

\subsection{Measurement protocol}

A 12-mm-diameter transducer at $1 \mathrm{MHz}$ was used. Then, three successive measurements on each side of the cubic lattice specimens were performed. Before each measurement, a reference measurement in air was taken to get rid of the derivative of the system.

\subsection{Results}

The measurements (several single points) performed at different distances from the specimens are display in Figs. 10, 11, and 12.
Figure 10 highlights that the measurement method used is repeatable as well as the AM process used to fabricate the lattice specimens.

Figure 11 shows that the measurement method enables to distinguish differences between the surfaces of the lattice specimen. The base surface is the one which was directly in contact with the AM platform which significate that it had to be mechanically detached from it.

Figure 12 demonstrates that the measurement method enables to distinguish differences between two percentages of cells in lattice specimens and confirms that higher percentage of cells, i.e., less metal, has a lower electrical conductivity (the slope of the quasi-linear curve of $79 \%$ cells is higher).
Fig. 11 Complex impedance measurements of the different faces of a lattice specimen (E1) with a theoretical percentage of cells of $77 \%$ using an eddy current system

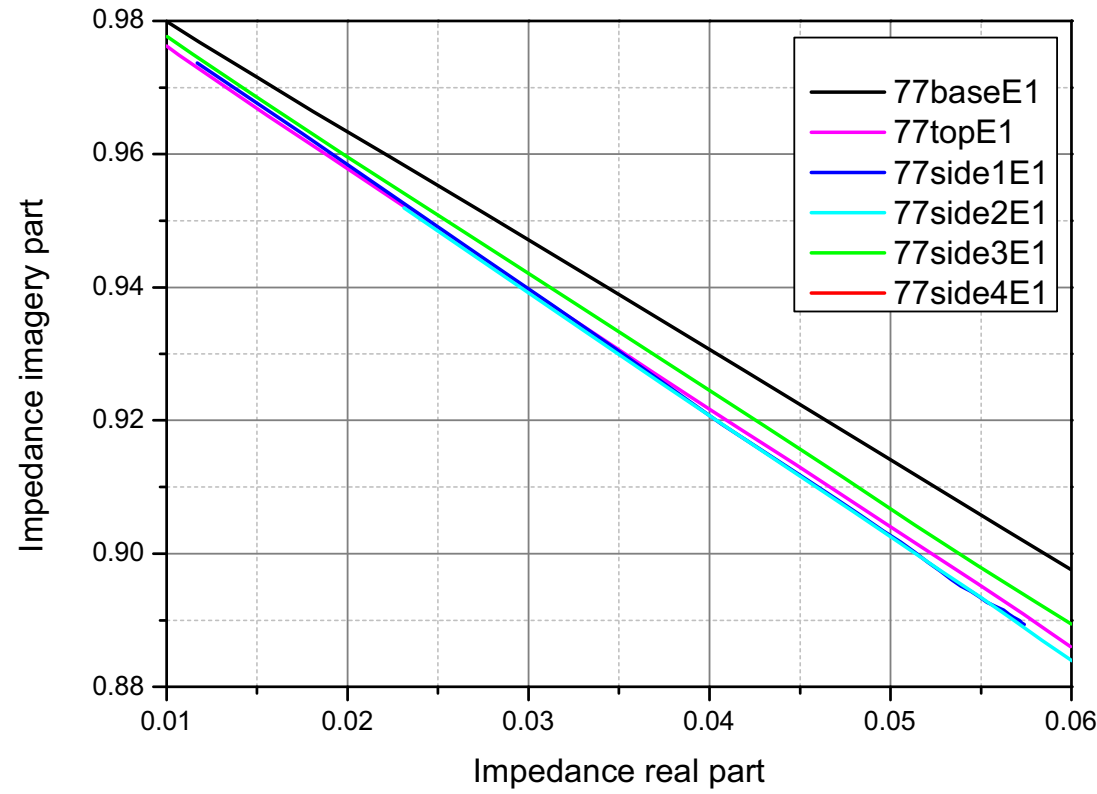


Fig. 12 Three successive complex impedance measurements of two different theoretical percentage of cells of 77 and $79 \%$ using an eddy current system

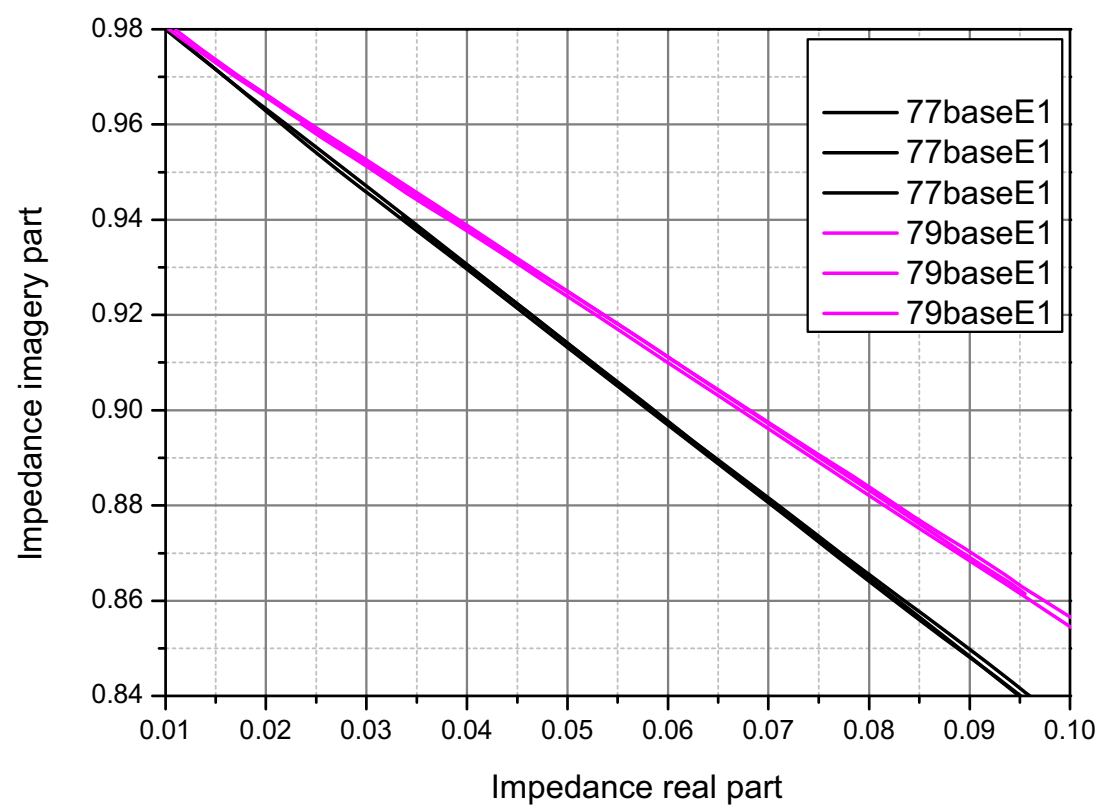

\subsection{Benefits and limitations of the method}

The multi-frequency eddy current instrument Z-Scope*7 from Sciensoria enables to measure the apparent electrical conductivity of the samples which can be used to characterise a dense structure as well as a lattice one. The roughness of the sample surface does not prevent measurement as the probe is not directly in contact with the sample and even the method enables to distinguish two different surface textures as well as two different pore size lattice structures. Furthermore, comparing similar parts fabricated during the same AM process and similar parts fabricated in different AM processes enables to study the repeatability and the reproducibility, respectively, of the AM processes. Finally, the measurements are fast so the method is convenient for routine control. However, the measurements are limited to a depth close to the surface so does not characterise the volume in its entirety and is not appropriate for parts less than $1 \mathrm{~cm}^{3}$ in volume.

\section{C-scan ultrasound characterisation method}

\subsection{Measurement system}

The Eurosonic C-scan ultrasound characterisation method is presented in Fig. 13. It consists of a container full of water, a four axe $(\mathrm{x}, \mathrm{y}, \mathrm{z}, \theta)$ arm at the extremity of which is hang an emission/reception ultrasound transducer. A specific emission/reception electronic card is adapted to this transducer. The system is controlled by a dedicated
Fig. 13 Eurosonic C-scan ultrasound system used to characterise the quality of the studied parts

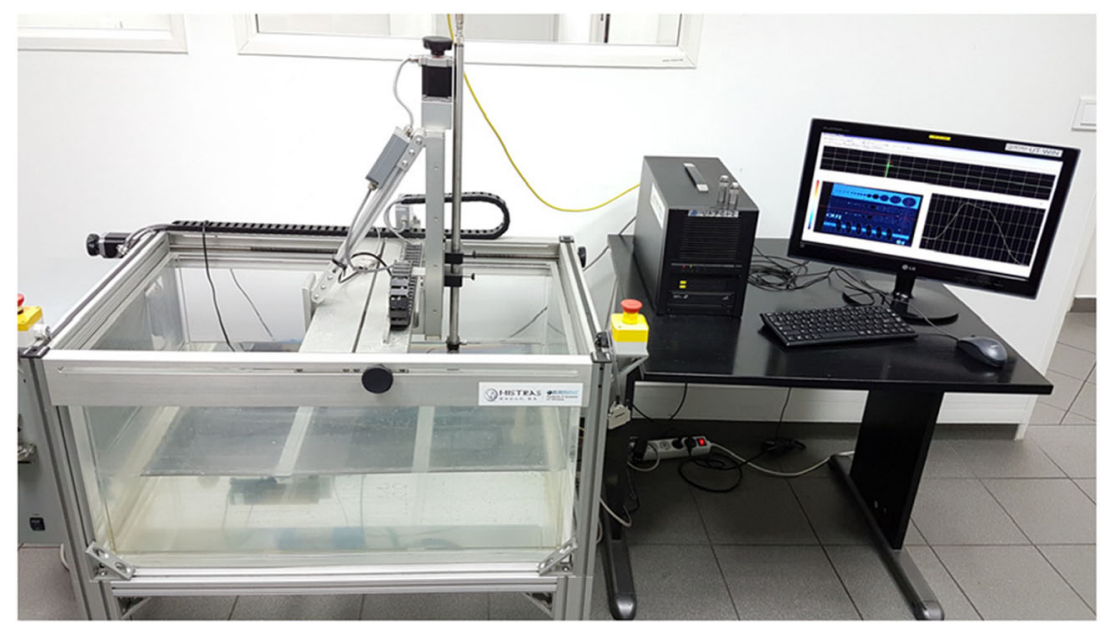



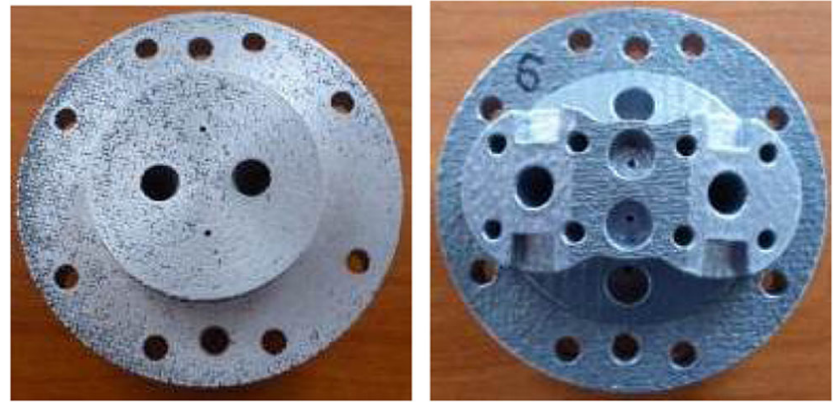

Fig. $14 \mathrm{AM}$ aluminium studied parts (left: top of the part relative to the scan, right: bottom of the part relative to the scan)

software on a computer. The software also displays the Ascan, B-scan, and C-scan and enables to go through the Cscan (4D C-scan).

\subsection{Measurement protocol}

Two different AM aluminium (Al) parts were characterised. These parts are presented in Figs. 14 and 16 (top). They were scanned using a $19-\mathrm{mm}$ spherical focused $20-\mathrm{MHz}$ transducer and a water column of $18 \mathrm{~mm}$. The spatial scan resolution is of $0.1 \mathrm{~mm}$.

\subsection{Results}

As this is presented in Figs. 15 and 16, we can clearly see the inside features of the parts on the C-scan ultrasound characterisation. In Fig. 16, even the grooves on the opposite side of the part are imaged (Fig. 16 bottom, red square on the right) as well as the spirals inside the hollow cylinder (Fig. 16 bottom, red square on the left).

\subsection{Benefits and limitations of the method}

The C-scan ultrasound method from Eurosonic provides 3D images of a sample surface but also of the inside features of a sample. From these 3D images, nondestructive quality controls can be performed as well as dimensional measurements. Consequently, the compliance with the part geometrical specifications can be checked. Furthermore, the measurements are much faster than with
Fig. 15 Ultrasound

characterisation of an $\mathrm{Al}$ part (top) and numerical model of this part (bottom)

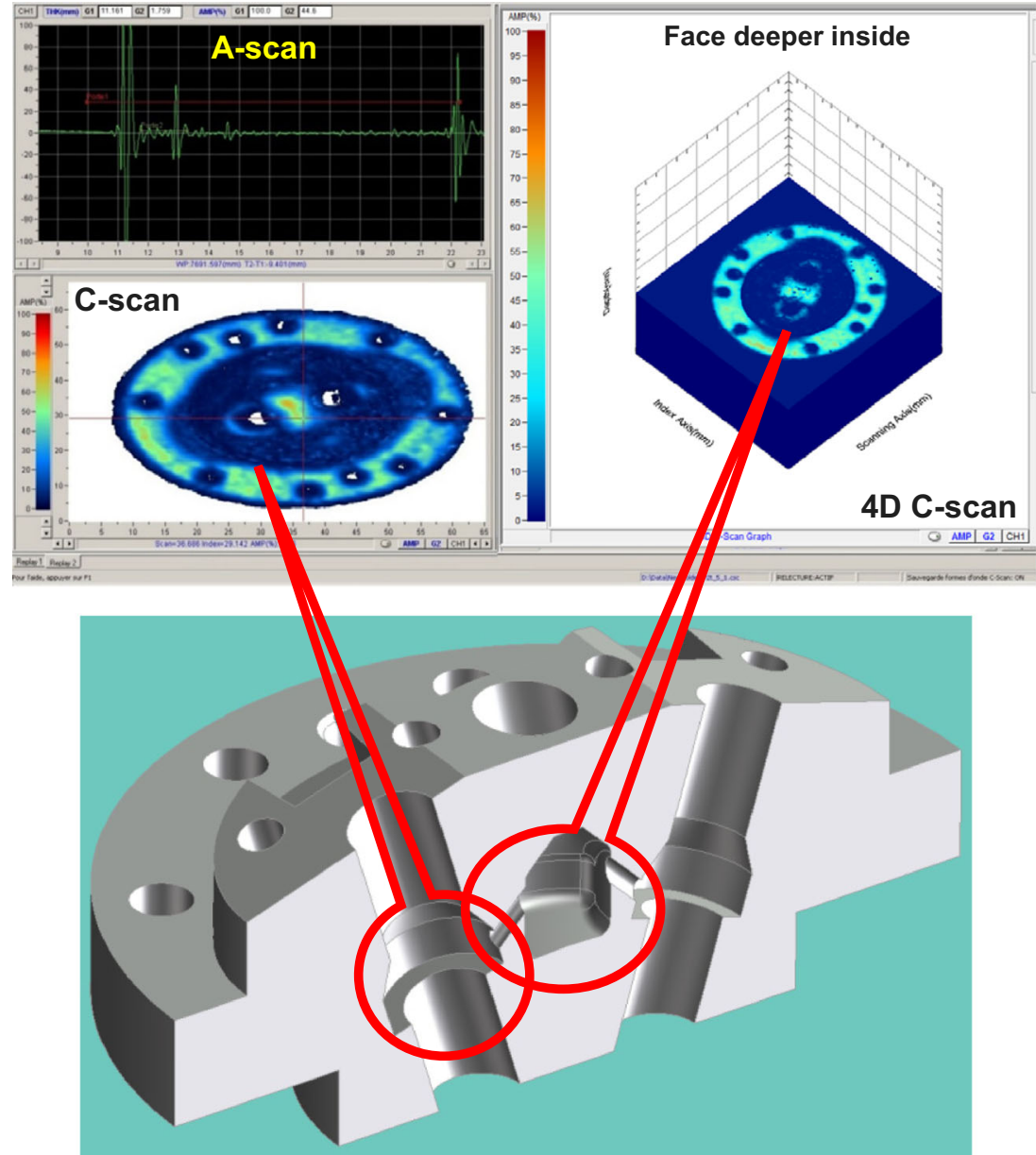




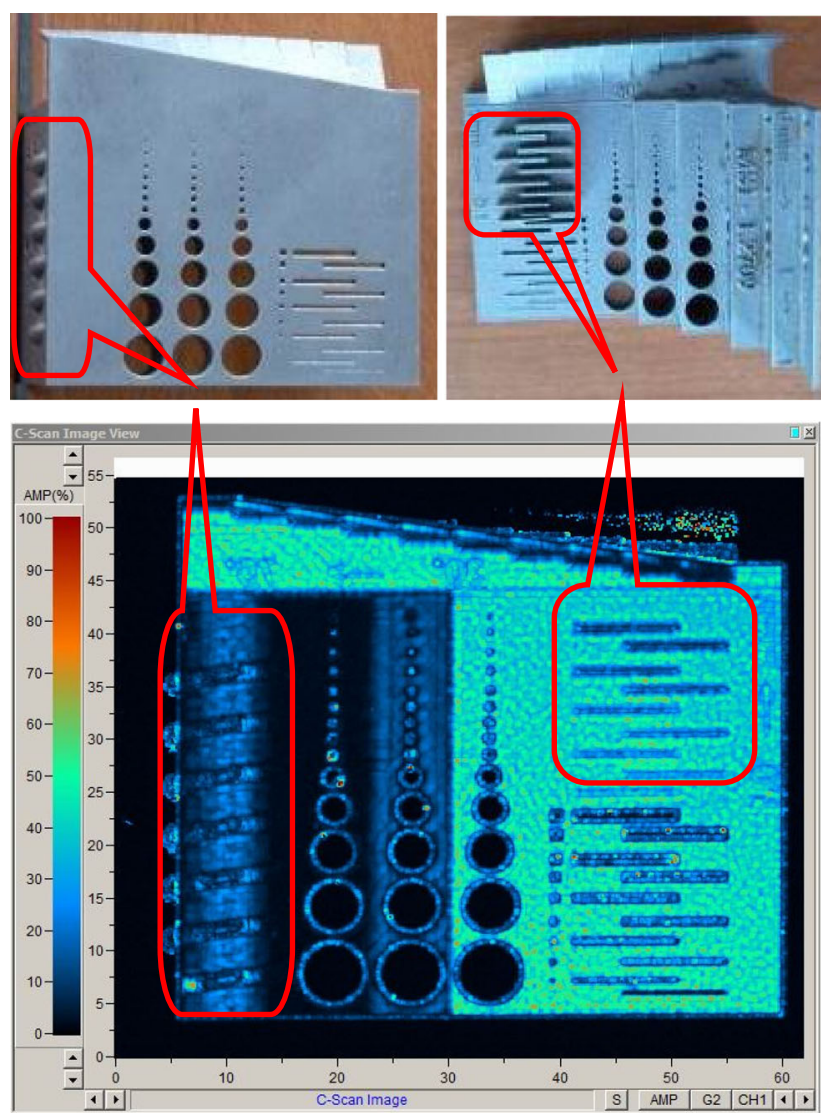

Fig. 16 Al part fabricated by Initial (top left: as it was scanned, top right: opposite side) and ultrasound characterisation of this part (bottom)

the X-ray tomography (XCT) so the method is convenient for routine control. However, the method is not suitable for complex geometry parts and less accurate than XCT.

\section{Conclusion}

Different volumetric methods were investigated in order to determine their capabilities for the characterisation of additively manufactured parts. First of all, an Archimedes' and pycnometric methods were investigated to perform density measurements on dense and lattice structures. Then, an eddy current method to determine the electrical conductivity via complex impedance measurements of lattice structures was presented. Finally, a C-scan ultrasound method for the characterisation of dense parts was explored. For each of these methods, the advantages and limitations were underlined.

Funding information The work was supported by the project "MetAMMI" and has received funding from the EMPIR programme co-financed by the Participating States and from the European Union's Horizon 2020 research and innovation programme.

Open Access This article is distributed under the terms of the Creative Commons Attribution 4.0 International License (http:// creativecommons.org/licenses/by/4.0/), which permits unrestricted use, distribution, and reproduction in any medium, provided you give appropriate credit to the original author(s) and the source, provide a link to the Creative Commons license, and indicate if changes were made.

\section{References}

1. Todorov E, Spencer R, Gleeson S, Jamshidinia M, Kelly SM (2014) Project 1: nondestructive evaluation (NDE) of complex metallic additive manufactured (AM) structures, AFRL-RX-WP-TR-2014-0162

2. Hermanek P, Carmignato S (2017) Porosity measurements by X-ray computed tomography: accuracy evaluation using a calibrated object. Precis Eng 49:377-387

3. Obaton A-F, Fain J, Djemaï M, Meinel D, Léonard F, Mahé E, Lécuelle B, Fouchet J-J, Bruno G (2017) In vivo XCT bone characterization of lattice structured implants fabricated by additive manufacturing: a case report. Heliyon 3. https://doi.org/10.1016/j. heliyon.2017.e00374

4. Gapinskia B, Janicki P, Marciniak-Podsadna L, Jakubowicz M (2016) Application of the computed tomography to control parts made on additive manufacturing process. Procedia Eng 149:105-121 